\title{
Effect of Local and Non-Local Kernels on Heat Transfer of Mixed Convection Flow of the Maxwell Fluid
}

\author{
M. B. Riaz $\mathbb{D}^{1,2}$ A. Atangana, ${ }^{2}$ Maryam Asgir, ${ }^{3,4}$ Muhammad Altaf Khan ${ }^{\mathbb{D}}{ }^{2}$ \\ and Hafte Amsalu Kahsay $\mathbb{1}^{5}$ \\ ${ }^{1}$ Department of Mathematics, University of Management and Technology, Lahore 54770, Pakistan \\ ${ }^{2}$ Institute for Groundwater Studies, University of the Free State, Bloemfontein 9301, South Africa \\ ${ }^{3}$ Department of Mathematics, Government College University, Lahore 54000, Pakistan \\ ${ }^{4}$ Department of Mathematics, Riphah International University, Lahore, Pakistan \\ ${ }^{5}$ Department of Mathematics, College of Natural Science, Wollo University, Dessie, Ethiopia
}

Correspondence should be addressed to Hafte Amsalu Kahsay; yohanahafte@gmail.com

Received 11 March 2021; Revised 26 April 2021; Accepted 24 May 2021; Published 12 June 2021

Academic Editor: Erhan Set

Copyright ( $\odot 2021$ M. B. Riaz et al. This is an open access article distributed under the Creative Commons Attribution License, which permits unrestricted use, distribution, and reproduction in any medium, provided the original work is properly cited.

\begin{abstract}
The heat transfer study of mixed convection flow of the Maxwell fluid is carried out here. The fluid flow is demonstrated by the system of coupled partial differential equations in the dimensionless form firstly. Then, its fractional form is developed by using the new definition of the noninteger-order derivative with the singular kernel (Caputo/C) and nonsingular kernels (Caputo-Fabrizio/CF and Atangana-Baleanu (nonlocal)/ABC). The hybrid-form solutions are obtained by applying the Laplace transform, and for the inverse Laplace transform, the problem is tackled by the numerical algorithms of Stehfest and Tzou. The C, $\mathrm{CF}$, and $\mathrm{ABC}$ solution comparison under the effects of considered different parameters is depicted. The physical aspects of the considered problem are well explained by $\mathrm{C}, \mathrm{CF}$, and $\mathrm{ABC}$ in comparison to the integer-order derivative due to its memory effects. Furthermore, the best fit model to explain the memory effects of velocity is CF. The solutions for the Newtonian fluid and ordinary Maxwell fluid are considered as a special case and found in the literature.
\end{abstract}

\section{Introduction}

The combination of natural and forced convection is called mixed convection. This phenomenon gained a lot of popularity in recent years due to its vast applications in many fields of engineering, such as nuclear, chemical, food, aerospace, electrical, fluid dynamics, and astrophysics. The combined convection process comes into reality when free or forced convection alone is not enough to describe the heat transfer process properly. Many diverse significant effects of mixed convection in the heat transfer phenomenon were seen in [1-4]. Integer-order derivatives are used widely to model real-world problems mathematically. The nonlocal nature of the noninteger-order derivative makes it a more efficient tool as compared to the integer-order derivative. The property of the noninteger derivative to get a better insight of the rheological and hereditary properties of the material under consideration makes it more valuable. It has become essential in modeling the description of many complex dynamics in the field of industries, engineering, technology, etc.

Non-Newtonian fluids are more important as compared to the viscous and Newtonian fluids because they depict the complex phenomenon of real-life problems. Mostly, the problems that exist in engineering and industries exhibit a nonlinear relationship. Non-Newtonian fluids connect the shear stress and shear strain in a nonlinear relationship. This nonlinear relationship of the shear stress and shear strain makes the non-Newtonian fluid flow more subtle. Maxwell fluid is one of the widely studied non-Newtonian fluids due to its diversity and rheological properties [5]. The nature of the viscoelastic fluids is best described by the noninteger/fractional derivative due to the history of fluid flow. Bagley and 
Torvik [6] and Germnat [7] were pioneers who introduced the fractional approach in the viscoelastic fluids. The characteristics of the viscoelastic fluids were investigated by Mainardi and Spada [8] via a fractional approach, and they found that the results obtained were in a good settlement with the data given for the experiment. Caputo, Caputo-Fabrizio, and Atangana-Baleanu have introduced the definition of the fractional derivative which has been widely used in solving many problems of fluid dynamics and other engineering and science disciplines.

The flow properties of the fractional Maxwell fluid between two parallel plates were investigated by Haito and Mingyu [9]. They obtained the solution of the pressure gradient by finite Fourier cosine and Laplace transform. Jamil [10] explored the analytical solutions of the fractionalized Maxwell fluid flowing past on a vertical plate with slip effects. He developed the results for the ordinary Maxwell and viscous fluid as a special case. The problem of unsteady MHD flow of the Maxwell fluid in a porous medium was solved by Khan et al. [11]. They studied the motion of the fluid induced due to the sine or cosine oscillation of the plate and revealed the interesting behaviors of velocity and shear stress. Vieru and Zafar [12] examined the Couette flow of the Maxwell fluid with the slip condition. The results for velocity and shear stress due to the constant and sinusoidal motion of the plate are obtained by the Laplace integral transform.

The heat transfer phenomenon in the Maxwell fluid was studied by Imran et al. [13] and many others via a fractional approach. The analysis of heat transfer in the fractionalized Maxwell fluid under the effects of MHD and second-order slip effects was carried out by Aman et al. [14]. Semianalytical results for velocity and shear stress were obtained and compared graphically. Raza and Ullah [15] used the C and CF derivatives to study the heat transfer in the Maxwell fluid under the consideration of Newtonian heating. The comparison results revealed that the value of fluid temperature and velocity is lower in the C case. Merkin and Mahmood [16] studied the aspects of mixed convection of external flow of the surface which was chemical reactive and immersed in a porous medium. For vertical surfaces, such studies of mixed convective flow were conducted by Minto et al. [17]. The study of the mixed convection flow in a horizontal channel and under constant heat flux revealed interesting results which were demonstrated by Chou and Tsern [1]. Khan et al. [2] considered the exothermal and isothermal stretching disk with the phenomenon of mixed heat convection and presented the analytical results.

The purpose of this communication is to consider the Maxwell fluid over an oscillating vertical plate with constant wall temperature and analyze the phenomenon of mixed convective heat transfer via the fractional derivative definition of $\mathrm{C}, \mathrm{CF}$, and $\mathrm{ABC}$. Laplace transform is used to attain the solutions of the problem under deliberation, and these results of heat transfer analysis have much importance in the industry such as in cooling the circuits, radiator, heat exchanger, deicing system, solar panels, boilers, and many other home electric and industrial appliances.

\section{Mathematical Description of the Problem}

The incompressible Maxwell fluid unsteady mixed convective flow phenomenon on the oscillating vertical plate is considered here. Both fluid and plate are at rest at the start, and temperature is $T_{\infty}$.

As the time starts to increase, the plate starts to move in sinusoidal motion with the velocity $V=W_{0} H(t) \cos a t\left(W_{0}\right.$ is the amplitude of the motion, $H(t)$ is the unit step function, and $a$ represents the frequency of the plate oscillation) which results in the start of the flow of the Maxwell fluid. The temperature of the plate increases/decreases to a constant value $T_{w}$. With the increase in time, the velocity reaches zero and temperature to free stream temperature $T_{\infty}$. The heat transfer phenomenon of Maxwell fluid flow due to mixed convection along shear stress is described by the following PDEs [3]. The list of parameters is given in Table 1.

$$
\begin{aligned}
\rho\left(1+\lambda \frac{\partial}{\partial t}\right) \frac{\partial w(\eta, t)}{\partial t} & =\mu \frac{\partial^{2} w(\eta, t)}{\partial \eta^{2}}+\left(1+\lambda \frac{\partial}{\partial t}\right) \rho g \beta\left(T-T_{\infty}\right) \\
\left(1+\lambda \frac{\partial}{\partial t}\right) \tau(\eta, t) & =\mu \frac{\partial w(\eta, t)}{\partial \eta}, \\
\rho c_{p} \frac{\partial T(\eta, t)}{\partial t} & =k \frac{\partial^{2} T(\eta, t)}{\partial \eta^{2}}
\end{aligned}
$$


TABle 1: Nomenclature.

\begin{tabular}{lc}
\hline Symbol & Quantity \\
\hline$\rho$ & Fluid density \\
$\lambda$ & Relaxation time \\
$\mu$ & Viscosity (dynamic) \\
$\beta$ & Volumetric coefficient \\
$c_{p}$ & Specific heat \\
$g$ & Acceleration due to gravity \\
\hline
\end{tabular}

and the imposed initial and boundary conditions are

$w(\eta, t)=0, T(\eta, t)=T_{\infty}, \quad$ at $t=0$,

$w(\eta, t)=W_{0} H(t) \cos a t, T(\eta, t)=T_{w}, \quad$ at $\eta=0$,

$w(\eta, t)=0, T(\eta, t)=T_{\infty}, \quad$ as $\eta \longrightarrow \infty$.

The dimensionless relations are defined as

$$
\begin{aligned}
w^{*} & =\frac{w}{W_{0}}, \\
\eta^{*} & =\frac{\eta W_{0}}{v}, \\
t^{*} & =\frac{t W_{0}^{2}}{v}, \\
a^{*} & =\frac{a v}{W_{0}^{2}}, \\
\tau^{*} & =\frac{v \tau}{\mu W_{0}^{2}}, \\
\phi & =\frac{T-T_{\infty}}{T_{w}-T_{\infty}}, \\
\lambda^{*} & =\frac{\lambda W_{0}^{2}}{v}, \\
P_{r} & =\frac{\mu c_{p}}{k} . \\
G_{r} & =\frac{\nu g \beta\left(T_{w}-T_{\infty}\right)}{W_{0}^{3}},
\end{aligned}
$$

The dimensionless system of partial differential equations after dropping the " $*$ " notation is

$$
\begin{aligned}
\left(1+\lambda \frac{\partial}{\partial t}\right) \frac{\partial w(\eta, t)}{\partial t} & =\frac{\partial^{2} w(\eta, t)}{\partial \eta^{2}}+\left(1+\lambda \frac{\partial}{\partial t}\right) G_{r} \phi(\eta, t) \\
\left(1+\lambda \frac{\partial}{\partial t}\right) \tau(\eta, t) & =\frac{\partial w(\eta, t)}{\partial \eta}, \\
P_{r} \frac{\partial \phi(\eta, t)}{\partial t} & =\frac{\partial^{2} \phi(\eta, t)}{\partial \eta^{2}}
\end{aligned}
$$

and initial and boundary conditions in the dimensionless form are

$$
\begin{aligned}
& w(\eta, t)=0, \phi(\eta, t)=0, \quad t=0, \eta>0, \\
& w(\eta, t)=H(t) \cos a t, \phi(\eta, t)=1, \quad t>0, \eta=0, \\
& w(\eta, t)=0, \phi(\eta, t)=0, \quad \eta \longrightarrow \infty .
\end{aligned}
$$

\section{Preliminaries}

Caputo (C) fractional time derivative is defined as

$$
{ }^{C} D_{t}^{\alpha} g(\eta, t)=\frac{1}{\Gamma(1-\alpha)} \int_{0}^{t}(t-\xi)^{-\alpha} g^{\prime}(\eta, \xi) \mathrm{d} \xi, \quad \alpha \in(0,1),
$$

and its Laplace transform

$$
L\left({ }^{C} D_{t}^{\alpha} g(\eta, t)\right)=s^{\alpha} L(g(\eta, t))-s^{\alpha-1} g(\eta, 0) .
$$

Caputo-Fabrizio (CF) fractional time derivative is

$$
{ }^{C F} D_{t}^{\alpha} g(\eta, t)=\frac{1}{1-\alpha} \int_{0}^{t} e^{((-\alpha(t-\xi)) /(1-\alpha))} g^{\prime}(\eta, \xi) \mathrm{d} \xi, \quad \alpha \in(0,1),
$$

with the Laplace transform defined as

$$
L\left({ }^{C F} D_{t}^{\alpha} g(\eta, t)\right)=\frac{s L(g(\eta, t))-g(\eta, 0)}{s(1-\alpha)+\alpha} .
$$

Atangana-Baleanu $(\mathrm{ABC})$ time derivative is defined as

$$
{ }^{C} D_{t}^{\alpha} g(\eta, t)=\frac{1}{1-\alpha} \int_{0}^{t} E_{\alpha}\left(\frac{-\alpha(t-\xi)^{\alpha}}{1-\alpha}\right) g^{\prime}(\eta, \xi) \mathrm{d} \xi, \quad \alpha \in(0,1),
$$

and its Laplace transform is defined as

$$
L\left({ }^{A B C} D_{t}^{\alpha} g(\eta, t)\right)=\frac{s^{\alpha} L(g(\eta, t))-s^{\alpha-1} g(\eta, 0)}{s^{\alpha}(1-\alpha)+\alpha} .
$$

\section{Fractional Formulation of the Problem}

\subsection{Caputo Time Fractional Model}

$$
\begin{aligned}
\left(1+\lambda^{C} D_{t}^{\alpha}\right) \frac{\partial w(\eta, t)}{\partial t} & =\frac{\partial^{2} w(\eta, t)}{\partial \eta^{2}}+\left(1+\lambda^{C} D_{t}^{\alpha}\right) G_{r} \phi(\eta, t) \\
\left(1+\lambda^{C} D_{t}^{\alpha}\right) \tau(\eta, t) & =\frac{\partial w(\eta, t)}{\partial \eta} \\
P_{r}^{C} D_{t}^{\alpha} \phi(\eta, t) & =\frac{\partial^{2} \phi(\eta, t)}{\partial y^{2}}
\end{aligned}
$$


4.2. Caputo-Fabrizio Time Fractional Model

$$
\begin{aligned}
\left(1+\lambda^{C F} D_{t}^{\alpha}\right) \frac{\partial w(\eta, t)}{\partial t} & =\frac{\partial^{2} w(\eta, t)}{\partial \eta^{2}}+\left(1+\lambda^{C F} D_{t}^{\alpha}\right) G_{r} \phi(\eta, t) \\
\left(1+\lambda^{C F} D_{t}^{\alpha}\right) \tau(\eta, t) & =\frac{\partial w(\eta, t)}{\partial \eta} \\
P_{r}^{C F} D_{t}^{\alpha} \phi(\eta, t) & =\frac{\partial^{2} \phi(\eta, t)}{\partial \eta^{2}}
\end{aligned}
$$

\subsection{Atangana-Baleanu Time Fractional Model}

$$
\begin{aligned}
\left(1+\lambda^{A B C} D_{t}^{\alpha}\right) \frac{\partial w(\eta, t)}{\partial t} & =\frac{\partial^{2} w(\eta, t)}{\partial \eta^{2}}+\left(1+\lambda^{A B C} D_{t}^{\alpha}\right) G_{r} \phi(\eta, t) \\
\left(1+\lambda^{A B C} D_{t}^{\alpha}\right) \tau(\eta, t) & =\frac{\partial w(\eta, t)}{\partial \eta} \\
P_{r}{ }^{A B C} D_{t}^{\alpha} \phi(\eta, t) & =\frac{\partial^{2} \phi(\eta, t)}{\partial \eta^{2}}
\end{aligned}
$$

\section{Solutions for Temperature}

5.1. Caputo Sense. By implementing the Laplace transform on equation (16),

$$
\frac{\partial^{2} \bar{\phi}(\eta, s)}{\partial \eta^{2}}-P_{r} s^{\alpha} \bar{\phi}(\eta, s)=0
$$

and the solution of the above equation is given as

$$
\bar{\phi}(\eta, s)=\frac{1}{s} e^{-\eta \sqrt{P_{r} s^{\alpha}}} .
$$

5.2. Caputo-Fabrizio Sense. Laplace transform of equation (19) is

$$
\frac{\partial^{2} \bar{\phi}(\eta, s)}{\partial \eta^{2}}-\frac{b_{0} s}{s+\gamma} \bar{\phi}(\eta, s)=0
$$

The above differential equation solution after applying the transformed boundary conditions of $\phi(\eta, s)$ from (6) and (7) is

$$
\bar{\phi}(\eta, s)=\frac{1}{s} e^{-\eta \sqrt{\left(\left(b_{0} s\right) /(s+\gamma)\right)}},
$$

where $a_{0}=(1 /(1-\alpha)), b_{0}=P_{r} a_{0}$, and $\gamma=\alpha a_{0}$.

5.3. Atangana-Baleanu Sense. Applying the Laplace transform on equation (22),

$$
\frac{\partial^{2} \bar{\phi}(\eta, s)}{\partial \eta^{2}}-\frac{b_{0} s^{\alpha}}{s^{\alpha}+\gamma} \bar{\phi}(\eta, s)=0,
$$

and the Laplace solution of the above equation is

$$
\bar{\phi}(\eta, s)=\frac{1}{s} e^{-\eta \sqrt{\left(\left(b_{0} s^{\alpha}\right) /\left(s^{\alpha}+\gamma\right)\right)}},
$$

where $a_{0}=(1 /(1-\alpha)), b_{0}=P_{r} a_{0}$, and $\gamma=\alpha a_{0}$.

\section{Solutions for Velocity}

6.1. Caputo Sense. By taking the Laplace transform of equation (14) and substituting the value of $\bar{\phi}(\eta, s)$ from equation (24),

$$
\frac{\partial^{2} \bar{w}(\eta, s)}{\partial \eta^{2}}-\left(1+\lambda s^{\alpha}\right) s \bar{w}(\eta, s)=\frac{-G_{r}\left(1+\lambda s^{\alpha}\right)}{s} e^{-\eta \sqrt{P_{r} s^{\alpha}}}
$$

The solution of equation (29) after the use of the corresponding transformed boundary conditions from (6) and (7) is

$$
\bar{w}(\eta, s)=\frac{s}{s^{2}+a^{2}} e^{-\eta \sqrt{s\left(1+\lambda s^{\alpha}\right)}}+\frac{G_{r}\left(1+\lambda s^{\alpha}\right)}{\left(P_{r} s^{\alpha+1}-s^{2}\left(1+\lambda s^{\alpha}\right)\right)}\left(e^{-\eta \sqrt{s\left(1+\lambda s^{\alpha}\right)}}-e^{-\eta \sqrt{P_{r} s^{\alpha}}}\right) .
$$

6.2. Caputo-Fabrizio Sense. By taking the value of $\bar{\phi}(\eta, s)$ from equation (26), the transformed velocity equation becomes

$$
\frac{\partial^{2} \bar{w}(\eta, s)}{\partial \eta^{2}}-\left(\frac{c_{0} s+\gamma}{s+\gamma}\right) s \bar{w}(\eta, s)=-G_{r}\left(\frac{c_{0} s+\gamma}{s+\gamma}\right) \frac{e^{-\eta \sqrt{\left(\left(b_{0} s\right) /(s+\gamma)\right)}}}{s}
$$

The solution of the above equation is given as

$$
\bar{w}(\eta, s)=\frac{s}{s^{2}+a^{2}} e^{-\eta \sqrt{s\left(\left(c_{0} s+\gamma\right) /(s+\gamma)\right)}}+\frac{G_{r}\left(c_{0} s+\gamma\right)}{\left(b_{0} s^{2}-s^{2}\left(c_{0} s+\gamma\right)\right)}\left(e^{-\eta \sqrt{s\left(\left(c_{0} s+\gamma\right) /(s+\gamma)\right)}}-e^{-\eta \sqrt{\left(\left(b_{0} s\right) /(s+\gamma)\right)}}\right)
$$


where $a_{0}=(1 /(1-\alpha)), b_{0}=P_{r} a_{0}, c_{0}=1+\lambda a_{0}, \quad$ and $\gamma=$ $\alpha a_{0}$.
6.3. Atangana-Baleanu Sense. Imposing the Laplace transform on equation (20), with the substitution of the value of $\bar{\phi}(\eta, s)$ from equation (28), we get

$$
\frac{\partial^{2} \bar{w}(\eta, s)}{\partial \eta^{2}}-\left(\frac{c_{0} s^{\alpha}+\gamma}{s^{\alpha}+\gamma}\right) s \bar{w}(\eta, s)=-G_{r}\left(\frac{c_{0} s^{\alpha}+\gamma}{s^{\alpha}+\gamma}\right) \frac{e^{-\eta} \sqrt{\left(\left(b_{0} s^{\alpha}\right) /\left(s^{\alpha}+\gamma\right)\right)}}{s} .
$$

Solving the above equation, we get the solution

$$
\bar{w}(\eta, s)=\frac{s}{s^{2}+a^{2}} e^{-\eta \sqrt{s\left(\left(c_{0} s^{\alpha}+\gamma\right) /\left(s^{\alpha}+\gamma\right)\right)}}+\frac{G_{r}\left(c_{0} s^{\alpha}+\gamma\right)}{\left(b_{0} s^{\alpha+1}-s^{2}\left(c_{0} s^{\alpha}+\gamma\right)\right)}\left(e^{-\eta \sqrt{s\left(\left(c_{0} s^{\alpha}+\gamma\right) /\left(s^{\alpha}+\gamma\right)\right)}}-e^{-\eta \sqrt{\left(\left(b_{0} s^{\alpha}\right) /\left(s^{\alpha}+\gamma\right)\right)}}\right),
$$

where $a_{0}=(1 /(1-\alpha)), b_{0}=P_{r} a_{0}, \quad c_{0}=1+\lambda a_{0}$, and $\gamma=$ $\alpha a_{0}$.

\section{Solutions for Shear Stress}

7.1. Caputo Sense. The shear stress equation after applying the Laplace transform and simplification is

$$
\bar{\tau}(\eta, s)=\frac{1}{\left(1+\lambda s^{\alpha}\right)} \frac{\partial \bar{w}(\eta, s)}{\partial \eta} .
$$

Taking the derivative of velocity equation (30) with respect to $\eta$ and putting the value in equation (35), we get the expression for shear stress:

$$
\bar{w}(\eta, s)=\frac{s}{s^{2}+a^{2}}\left(-\sqrt{\frac{s}{\left(1+\lambda s^{\alpha}\right)}}\right) e^{-\eta \sqrt{s\left(1+\lambda s^{\alpha}\right)}}+\frac{G_{r}}{\left(P_{r} s^{\alpha+1}-s^{2}\left(1+\lambda s^{\alpha}\right)\right)}\left(\left(\sqrt{P_{r} s^{\alpha}}\right) e^{-\eta \sqrt{P_{r} s^{\alpha}}}-\left(\sqrt{s\left(1+\lambda s^{\alpha}\right)}\right) e^{-\eta \sqrt{s\left(1+\lambda s^{\alpha}\right)}}\right) .
$$

7.2. Caputo-Fabrizio Sense. The equation of the shear stress after employing the Laplace transform on equation (18) is

$$
\bar{\tau}(\eta, s)=\left(\frac{s+\gamma}{c_{0} s+\gamma}\right) \frac{\partial \bar{w}(\eta, s)}{\partial \eta}
$$

Taking the derivative of velocity equation (32) with respect to $\eta$ and putting the value in equation (37), we get the expression for shear stress:

$$
\begin{aligned}
\bar{w}(\eta, s)= & \frac{s}{s^{2}+a^{2}}\left(-\sqrt{s\left(\frac{s+\gamma}{c_{0} s+\gamma}\right)}\right) e^{-\eta \sqrt{s\left(\left(c_{0} s+\gamma\right) /(s+\gamma)\right)}}+\frac{G_{r}(s+\gamma)}{\left(b_{0} s^{2}-s^{2}\left(c_{0} s+\gamma\right)\right)}\left(\left(\sqrt{\frac{b_{0} s}{s+\gamma}}\right) e^{-\eta \sqrt{\left(\left(b_{0} s\right) /(s+\gamma)\right)}}\right. \\
& \left.-\left(\sqrt{s\left(\frac{c_{0} s+\gamma}{s+\gamma}\right)}\right) e^{-\eta \sqrt{s\left(\left(c_{0} s+\gamma\right) /(s+\gamma)\right)}}\right)
\end{aligned}
$$

where $a_{0}=(1 /(1-\alpha)), b_{0}=P_{r} a_{0}, c_{0}=1+\lambda a_{0}$, and $\gamma=$ $\alpha a_{0}$.

7.3. Atangana-Baleanu Sense. Applying the Laplace transform on equation (21) and simplifying give

$$
\bar{\tau}(\eta, s)=\left(\frac{s^{\alpha}+\gamma}{c_{0} s^{\alpha}+\gamma}\right) \frac{\partial \bar{w}(\eta, s)}{\partial \eta} .
$$

The solution of the shear stress after substituting the derivative of velocity equation (34) in the above equation is 


$$
\begin{aligned}
\bar{w}(\eta, s)= & \frac{s}{s^{2}+a^{2}}\left(-\sqrt{s\left(\frac{s^{\alpha}+\gamma}{c_{0} s^{\alpha}+\gamma}\right)}\right) e^{-\eta \sqrt{s\left(\left(c_{0} s^{\alpha}+\gamma\right) /\left(s^{\alpha}+\gamma\right)\right)}} \\
& +\frac{G_{r}\left(c_{0} s^{\alpha}+\gamma\right)}{\left(b_{0} s^{\alpha+1}-s^{2}\left(c_{0} s^{\alpha}+\gamma\right)\right)}\left(\left(\sqrt{\frac{b_{0} s^{\alpha}}{s^{\alpha}+\gamma}}\right) e^{-\eta \sqrt{\left(b_{0} s^{\alpha}\right) /\left(s^{\alpha}+\gamma\right)}}-\left(\sqrt{s\left(\frac{c_{0} s^{\alpha}+\gamma}{s^{\alpha}+\gamma}\right)}\right) e^{-\eta \sqrt{s\left(\left(c_{0} s^{\alpha}+\gamma\right) /\left(s^{\alpha}+\gamma\right)\right)}}\right)
\end{aligned}
$$

where $a_{0}=(1 /(1-\alpha)), b_{0}=P_{r} a_{0}, c_{0}=1+\lambda a_{0}$, and $\gamma=$ $\alpha a_{0}$.

Similarly, solutions for velocity, shear stress, and temperature for the ABC model were given by Abro et al. [18] $(M=0$ and $\Phi=0$ in Equation (2.8)). For the CF solution of velocity, temperature, and shear stress, we found the results in $[19,20]\left(\alpha_{2}=0\right.$ in Equation $\left.(7)\right)$.

The Laplace inverse transform for the solution of velocity and temperature is attained by applying Stehfest and Tzou numerical algorithms [21, 22].

Stehfest's algorithm is defined as

$$
\omega(\Psi, \tau)=\frac{\ln (2)}{\tau} \sum_{z=1}^{2 n} d_{z} \bar{\omega}\left(\Psi, \frac{z \ln (2)}{\tau}\right)
$$

where $n$ is a positive integer and

$$
d_{z}=(-1)^{z+n} \sum_{i=[(z+1) / 2]}^{\min (z, n)} \frac{i^{n}(2 i) !}{(n-i) ! i !(i-1) !(z-i) !(2 i-z) !},
$$

where the real number integer part is described by [.]. Tzou's numerical algorithm is defined as

$$
\bar{w}(\gamma, t)=\frac{e^{4.7}}{t}\left[\frac{1}{2} \bar{w}\left(\gamma, \frac{4.7}{t}\right)+\operatorname{Re}\left\{\sum_{l=1}^{N_{1}}(-1)^{l} \bar{w}\left(\gamma, \frac{4.7+z \pi i}{t}\right)\right\}\right],
$$

where $\operatorname{Re}($.$) is the real part, the imaginary part is i$, and $N_{1} \gg>1$ represents the natural number.

\section{Results and Discussion}

A comparative study of heat transfer in mixed convective flow of the Maxwell fluid with the noninteger-order approaches $\mathrm{C}, \mathrm{CF}$, and $\mathrm{ABC}$ is elaborated. To obtain the solutions of velocity, temperature, and shear stress via $\mathrm{C}, \mathrm{CF}$, and $\mathrm{ABC}$ fractional approaches, the Laplace transform is employed, and numerical algorithms are used to gain the solutions of the inverse Laplace transform. Effects of a fractional parameter, Prandtl number, and Grashof number on velocity, temperature, and shear stress are depicted graphically, and a comparison is made between three approaches to reveal the interesting results.

Figure 1 shows the physical insight of the fractional parameter on the temperature profile. Temperature increases with an increment in fractional parameter values in the $\mathrm{C}, \mathrm{CF}$, and $\mathrm{ABC}$ approach along with the variation of time. We see that, with the increase in time, the temperature increases and reaches a steady state rapidly in $\mathrm{CF}$ and $\mathrm{ABC}$. A slight difference is noticed in the result of $\mathrm{CF}$ and $\mathrm{ABC}$.

The Prandtl number $\operatorname{Pr}$ controls the thickness of the thermal boundary layer, resulting in a decrease in temperature with time variation as seen in Figure 2. We see a sharp decline in the temperature profile in the CF model, and it tends to the steady state slowly in comparison with $\mathrm{C}$ and $\mathrm{ABC}$.

The fractional parameter $\alpha$ controls the velocity of the fluid which is presented in Figure 3. As time varies from small to large, we see that the velocity becomes the increasing function of $\alpha$. The reason, $\alpha$ increases, boundary layer thickness increases, resulting in acceleration of the velocity. We see the highest velocity for CF. For $\alpha \longrightarrow 1$, we will recover the solution for integer-order derivatives which are already present in [3].

Figure 4 depicts the consequences of the Grashof number $\mathrm{Gr}$ on the velocity profile at varying time scale.

The graphical illustration depicts the velocity of fluid flow rises with the increase in Gr. The buoyancy forces take control over the viscous forces and result in accelerating the velocity. $\mathrm{CF}$ shows the highest velocity compared to $\mathrm{C}$ and $\mathrm{ABC}$.

The significant impact of $\operatorname{Pr}$ on the velocity profile is depicted in Figure 5. The fluid flow decreases with the Pr rise. $\operatorname{Pr}$ increases, and the thermal conductivity decreases, resulting in an increase in viscosity which slows down the fluid flow. Clearly, the CF model shows the highest velocity.

Figure 6 analyzes the influence of the Grashof number Gr on shear stress at different time scales. Shear stress increases with the increase in the Grashof number Gr near the boundary, and then there comes a critical point in $y$ where it starts to decline. The critical point moves further for $\mathrm{CF}$ as compared to $\mathrm{C}$, but it moves closer to the boundary in case of ABC.

To highlight the Pr behavior on shear stress, we observed Figure 7. The quite opposite behavior of $\mathrm{Pr}$ is observed compared to Gr. The velocity decreases with an increase in time and Pr, and at the critical point, it changes its behavior in the opposite direction.

For $\lambda \longrightarrow 0$, the results for Newtonian fluids are recovered and found in [23]. The results of the comparison of Maxwell and Newtonian fluids for C, CF, and ABC are illustrated in Figure 8. 


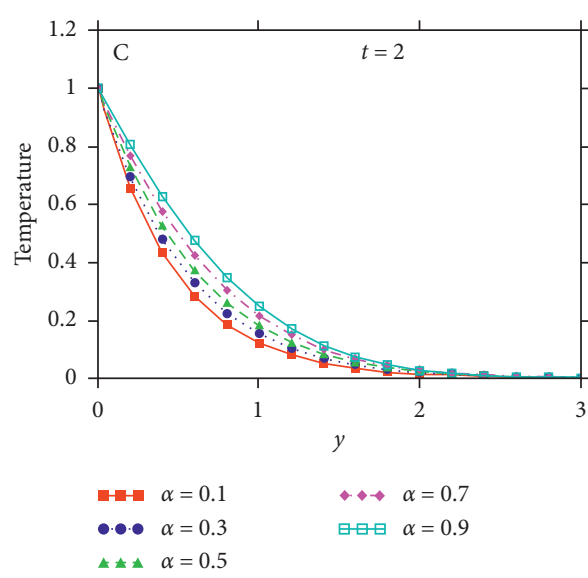

(a)

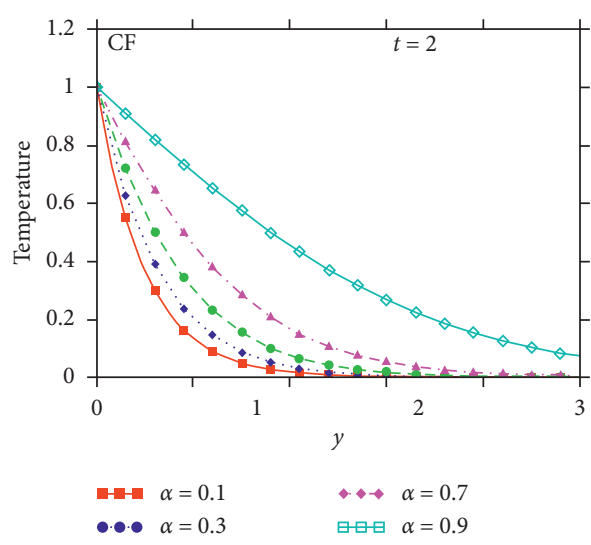

소숭 $\alpha=0.5$

(c)

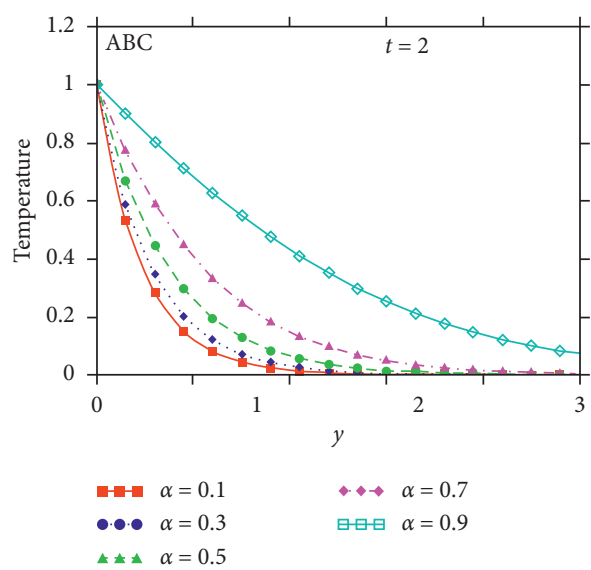

(e)

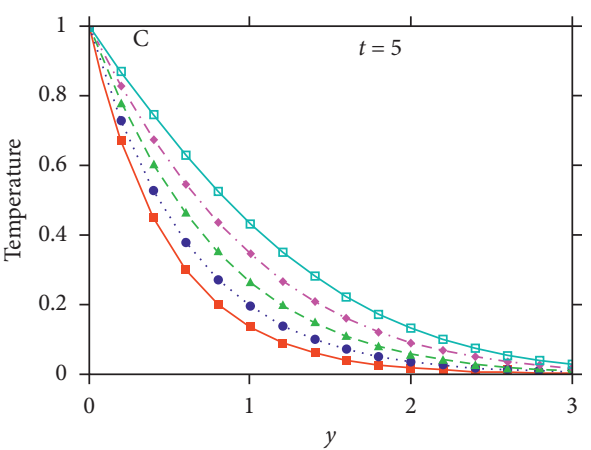

$\alpha=0.1 \quad \leftrightarrow \alpha=0.7$

$\bullet \alpha=0.3$

A $\alpha=0.5$

(b)

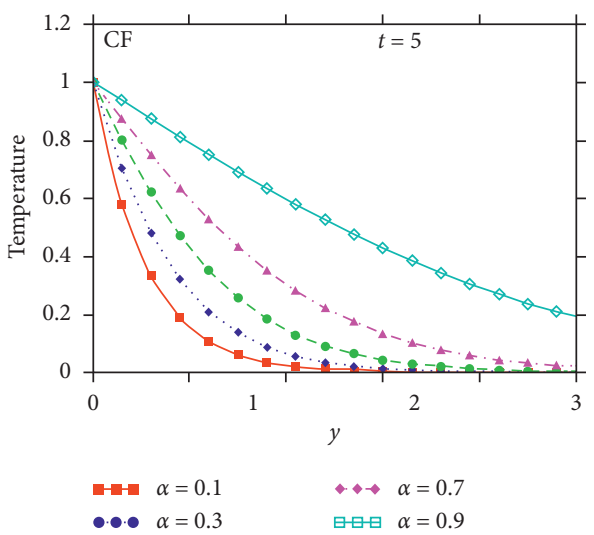

(d)

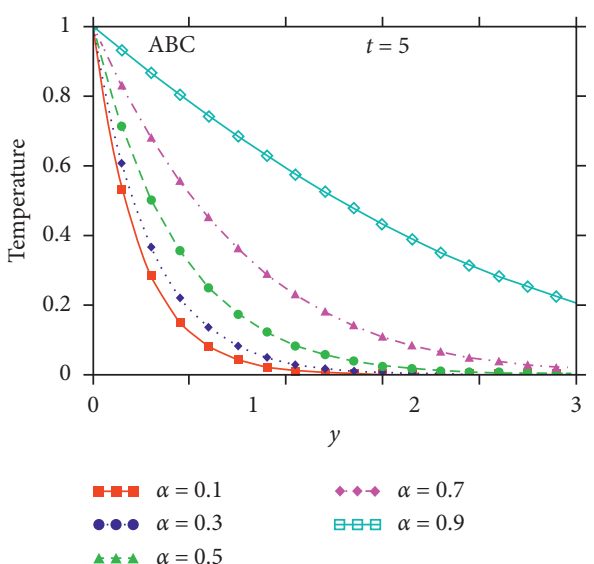

(f)

Figure 1: Temperature profile comparison via $\mathrm{C}, \mathrm{CF}$, and $\mathrm{ABC}$ with the variation of fractional parameter $\alpha$, with $\operatorname{Pr}=5$. 

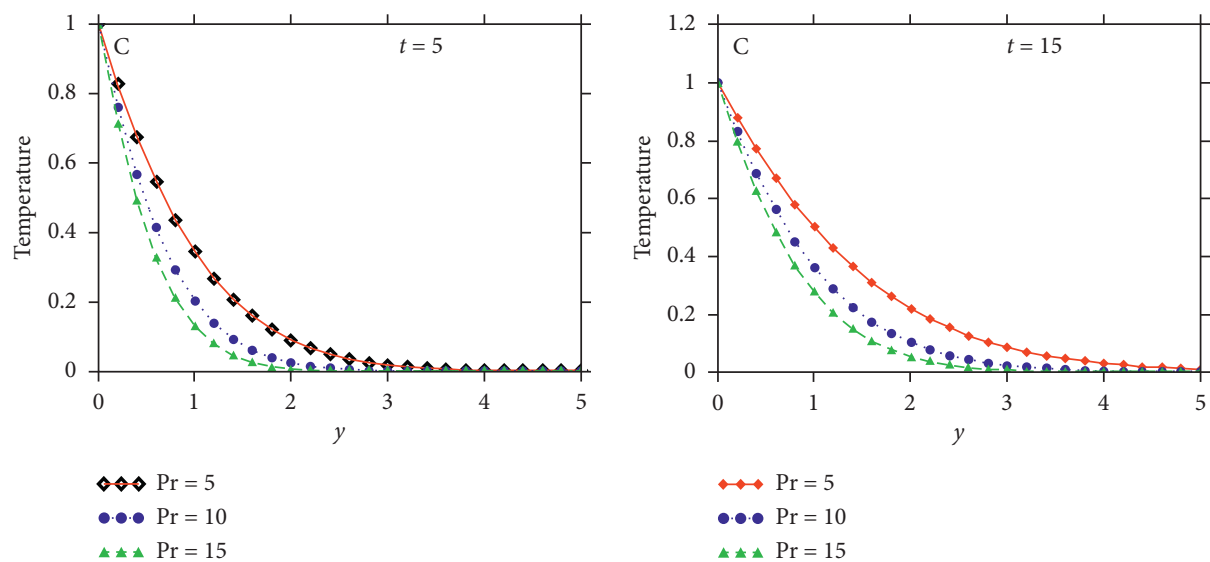

(a)

(b)
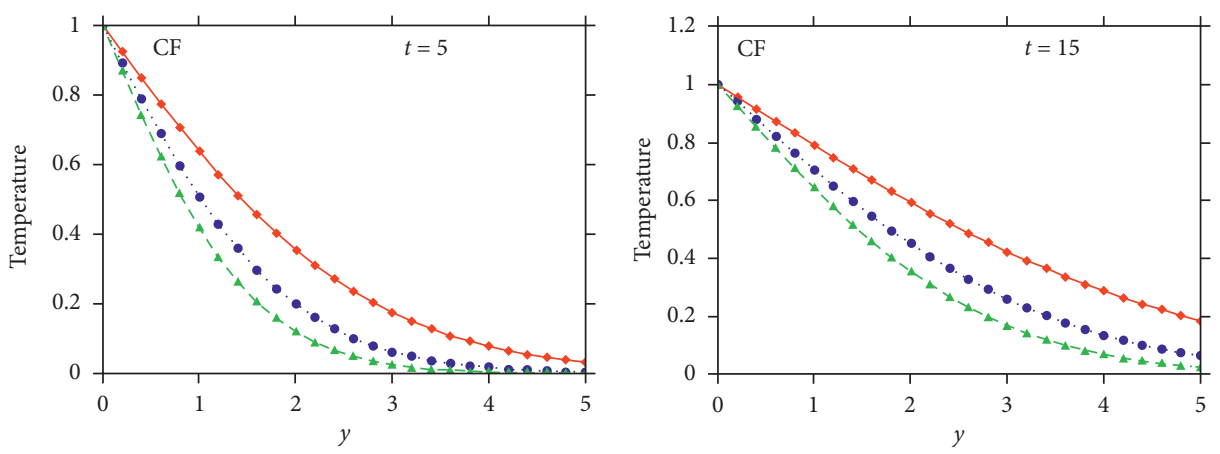

$$
\begin{aligned}
\because \operatorname{Pr} & =5 \\
\operatorname{Pr} & =10
\end{aligned}
$$

4. $\operatorname{Pr}=15$

$$
\begin{aligned}
& \leftrightarrow \operatorname{Pr}=5 \\
& \leftrightarrow \operatorname{Pr}=10 \\
& \leftrightarrow \operatorname{Pr}=15
\end{aligned}
$$

(c)

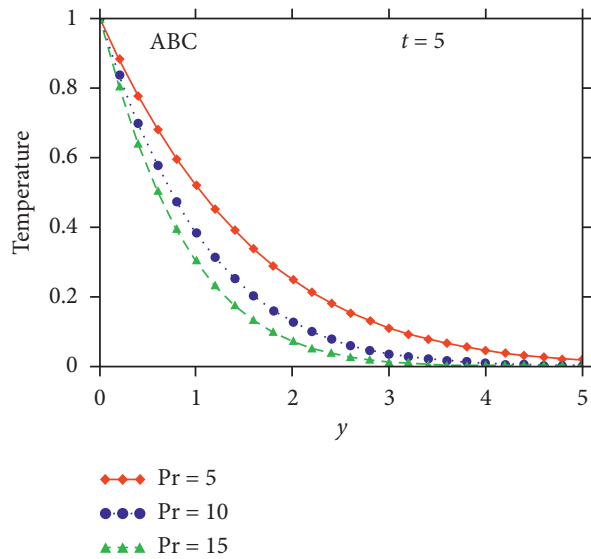

(e)

Figure 2: Temperature profile comparison via C, CF, and ABC with the Prandtl number variation, with $\alpha=0.7$. 


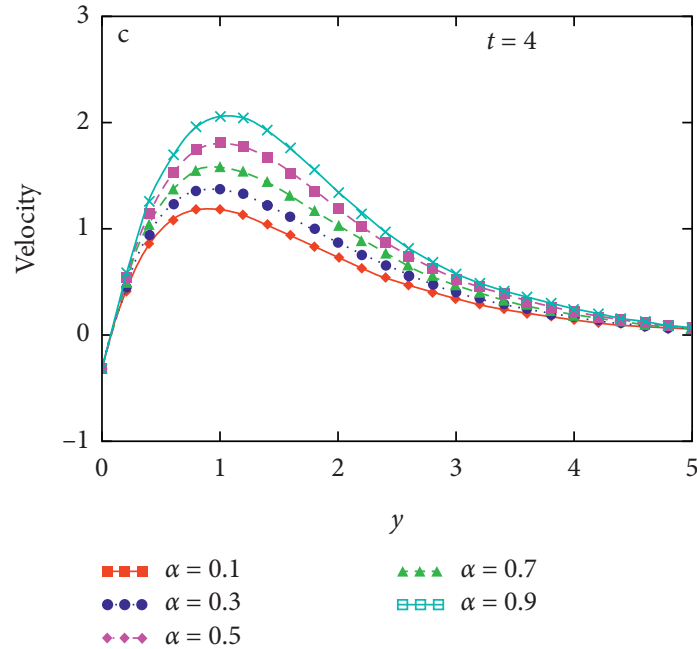

(a)

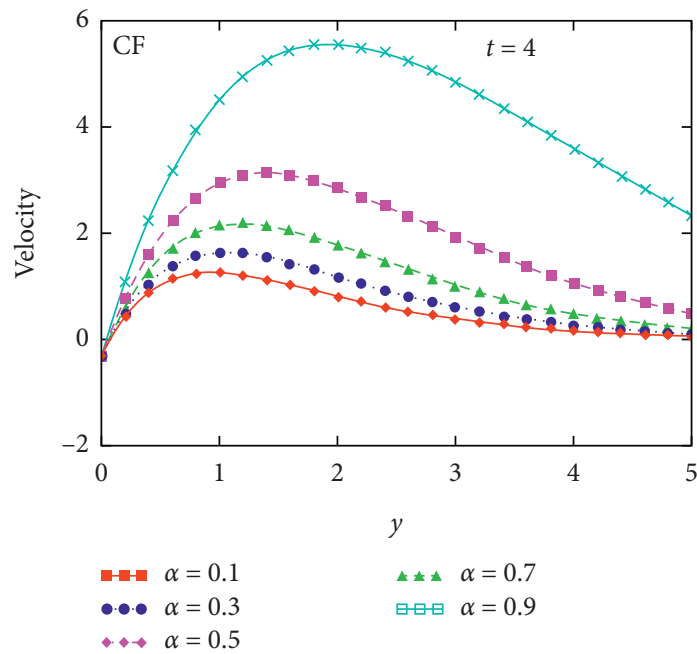

(c)

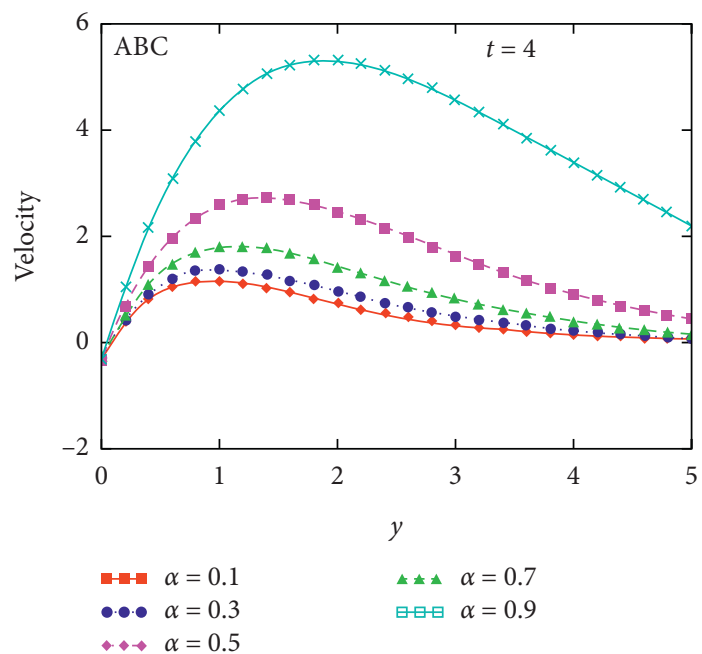

(e)

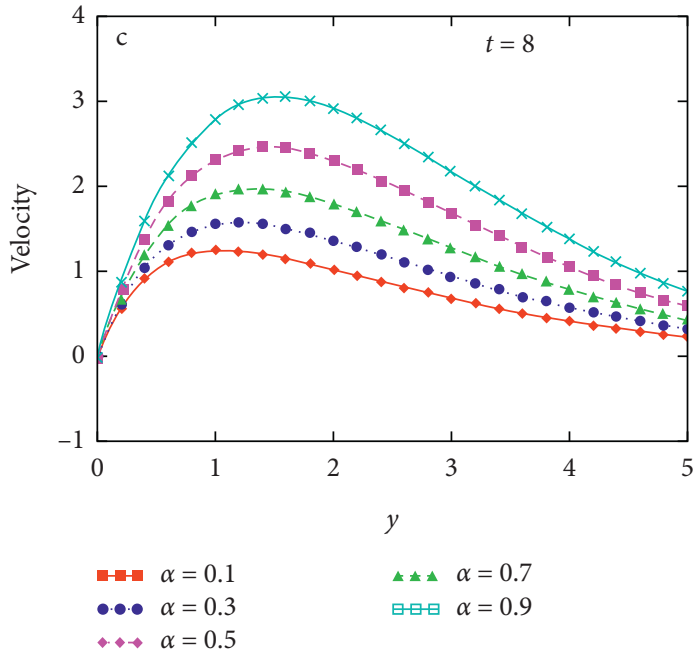

(b)

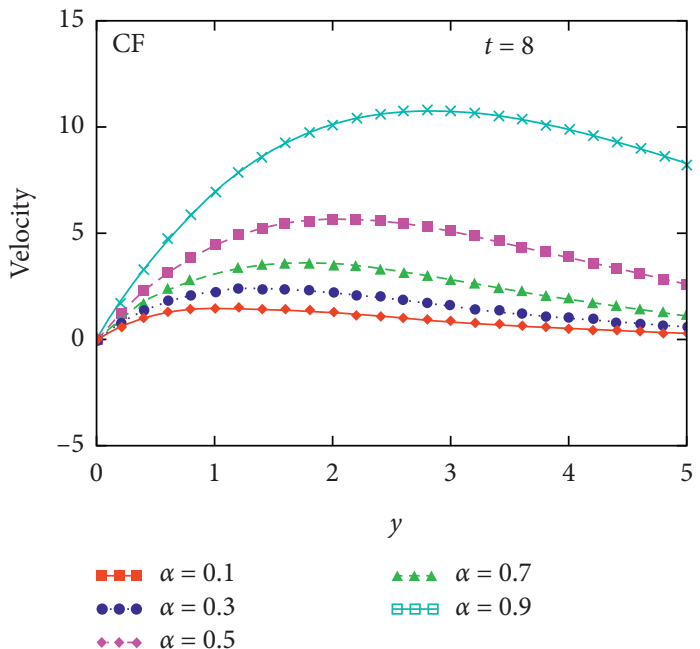

(d)

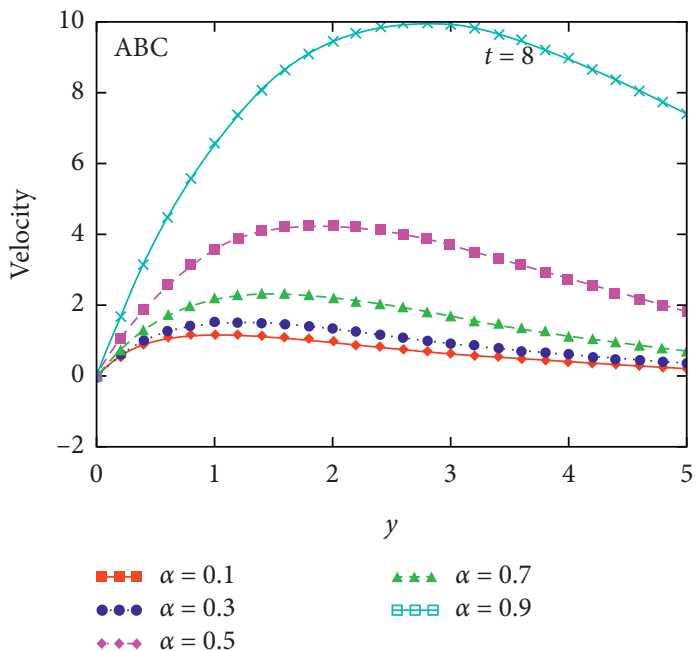

(f)

Figure 3: Velocity profile comparison via $\mathrm{C}, \mathrm{CF}$, and $\mathrm{ABC}$ with the variation of fractional parameter $\alpha$, and $\mathrm{Pr}=5$ and $\mathrm{Gr}=5$. 

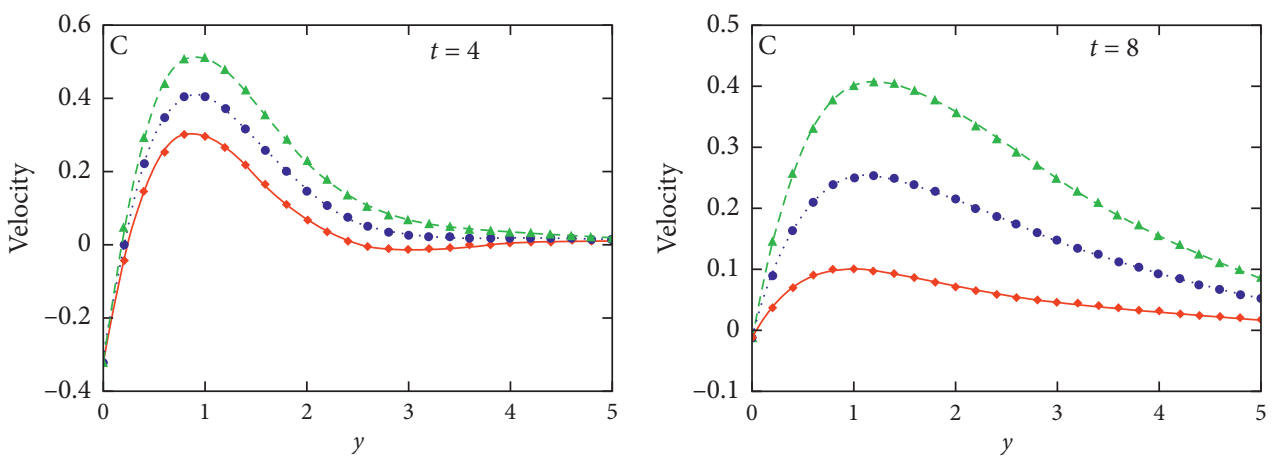

$\leadsto \mathrm{Gr}=0.2$

$\bullet \mathrm{Gr}=0.6$

A $\mathrm{A} \mathrm{Gr}=1.0$

$\leftrightarrow \mathrm{Gr}=0.2$

$\bullet \mathrm{Gr}=0.6$

A $A \mathrm{Gr}=1.0$

(a)

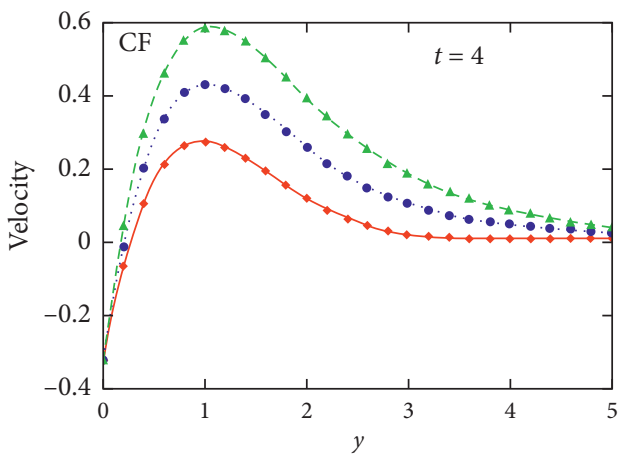

(b)

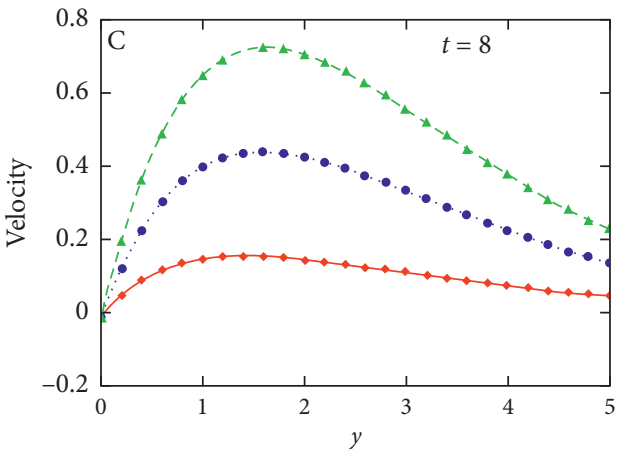

$\leftrightarrow \mathrm{Gr}=0.2$

$\leftrightarrow \mathrm{Gr}=0.2$

$\bullet \mathrm{Gr}=0.6$

$\star \star \star A G r=1.0$

(c)

(d)
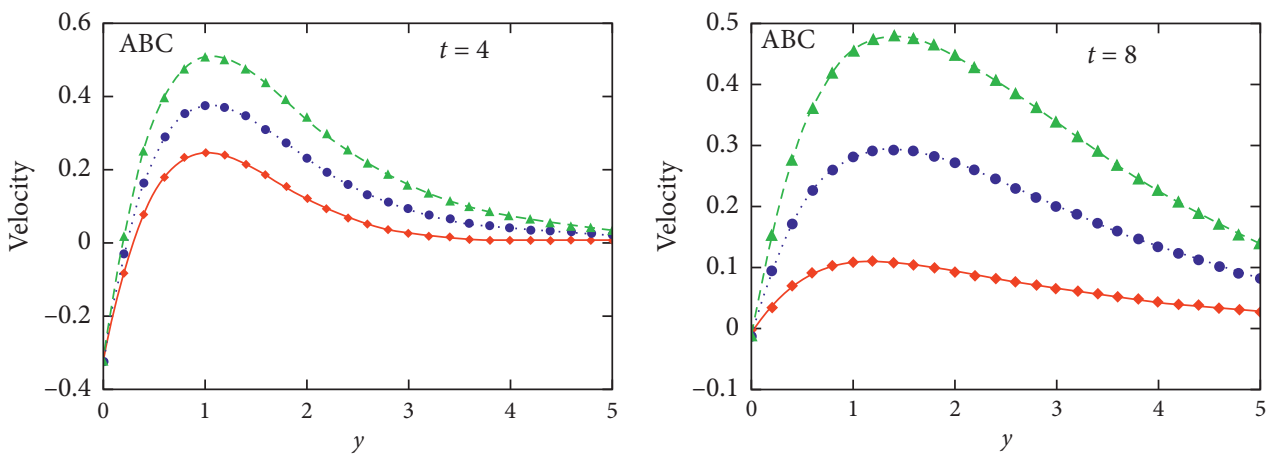

$\leftrightarrow \mathrm{Gr}=0.2$

$\leadsto \mathrm{Gr}=0.2$

••• $\mathrm{Gr}=0.6$

$\bullet \mathrm{Gr}=0.6$

A $\mathrm{A} \mathrm{Gr}=1.0$

(e)

(f)

Figure 4: Velocity profile comparison via $\mathrm{C}, \mathrm{CF}$, and $\mathrm{ABC}$ with the variation of the Grashof number $\mathrm{Gr}$, and $\operatorname{Pr}=5, a=2$, and $\lambda=0.7$. 

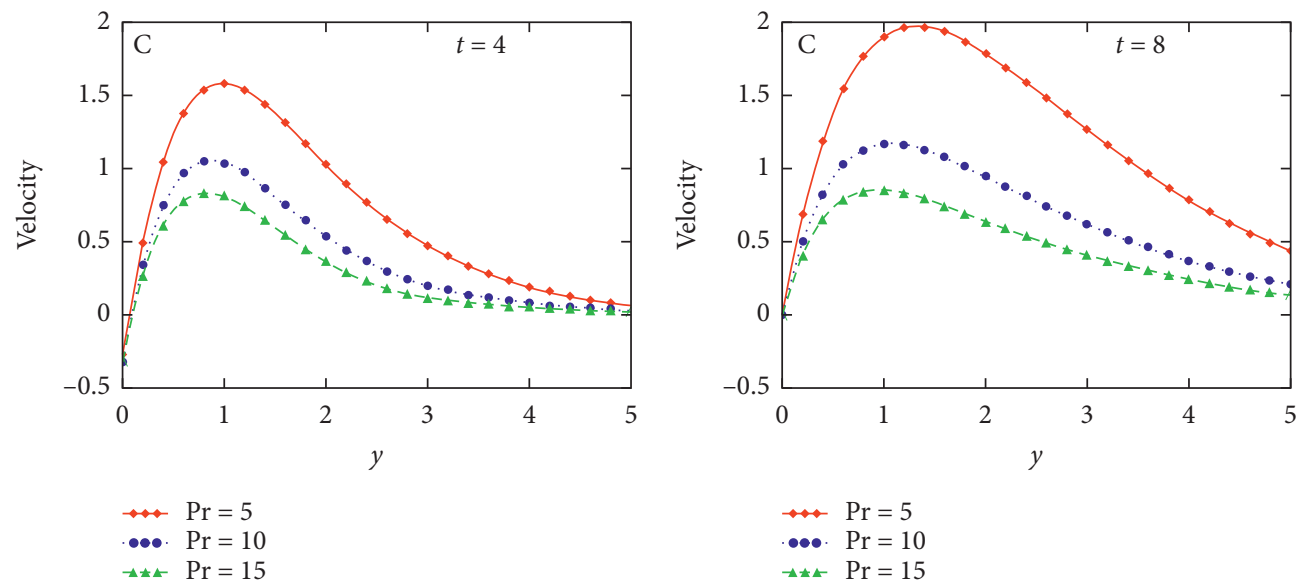

(a)

(b)
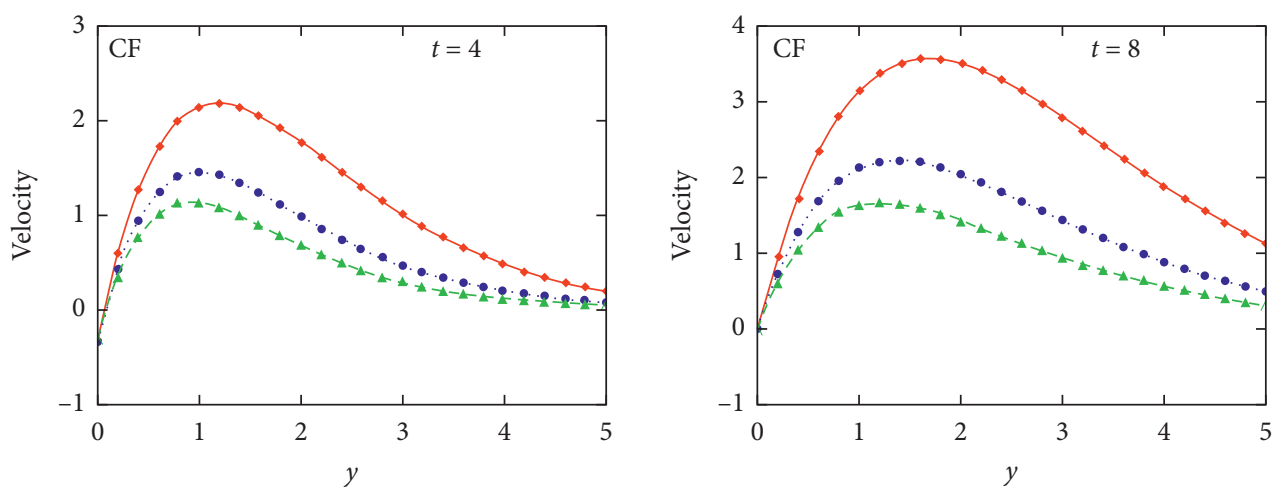

$\begin{aligned} \operatorname{Pr} & =5 \\ \operatorname{Pr} & =10\end{aligned}$

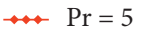

... $\operatorname{Pr}=10$

A声 $\operatorname{Pr}=15$

A\#亦 $\operatorname{Pr}=15$

(c)

(d)
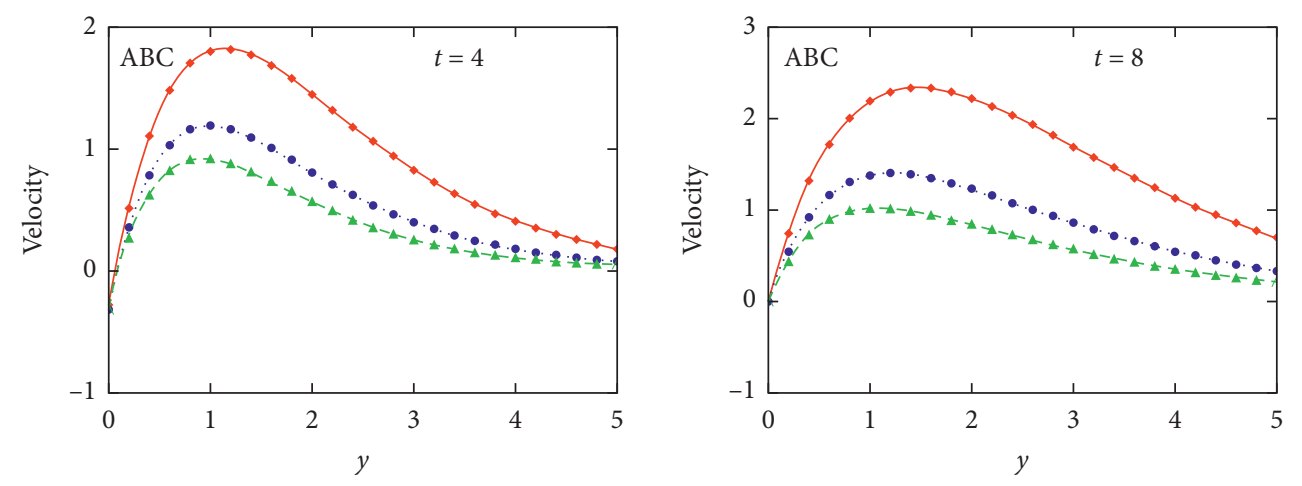

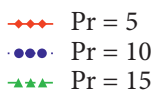

$\rightarrow \quad \operatorname{Pr}=5$

..e. $\operatorname{Pr}=10$

$\rightarrow$ A\#政 $\mathrm{Pr}=15$

(e)

(f)

Figure 5: Velocity profile comparison via C, CF, and $\mathrm{ABC}$ with the variation of the Prandtl number $\mathrm{Pr}$, and $\mathrm{Gr}=5, a=2$, and $\lambda=0.7$. 

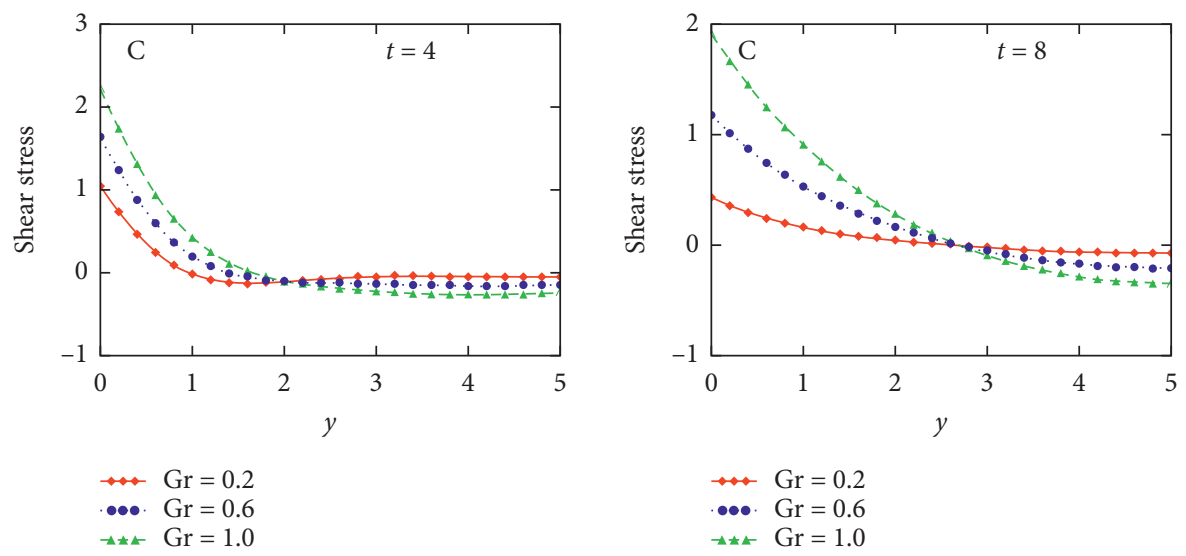

(a)
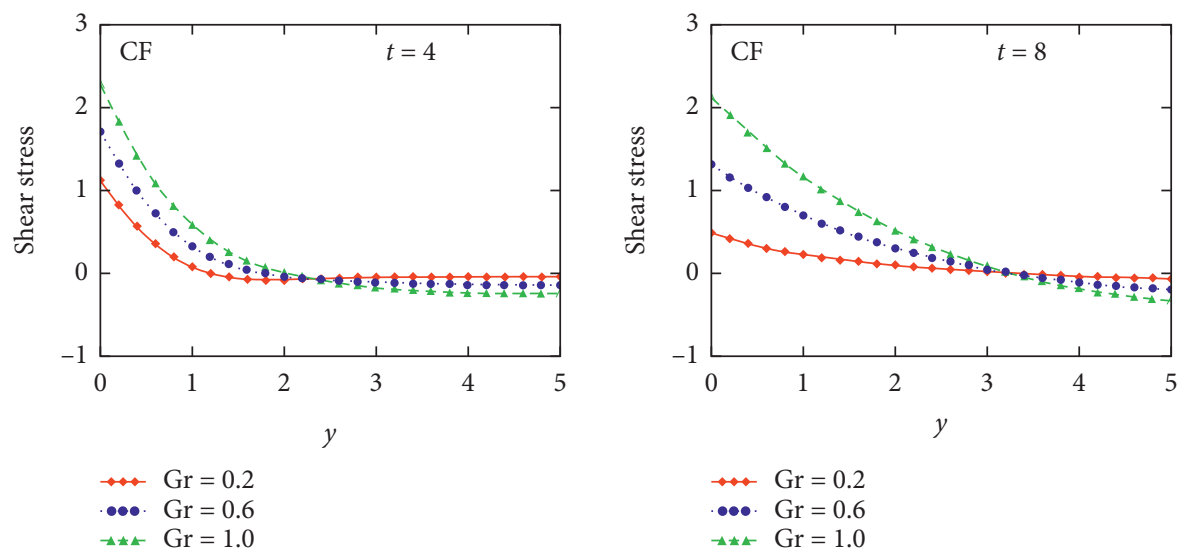

(c)

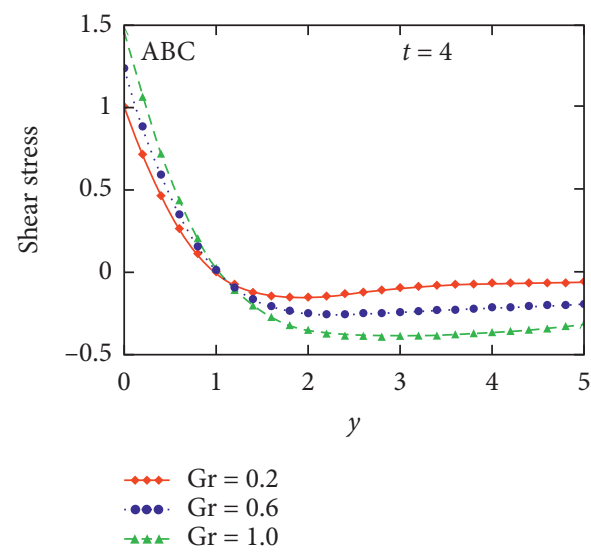

(e)

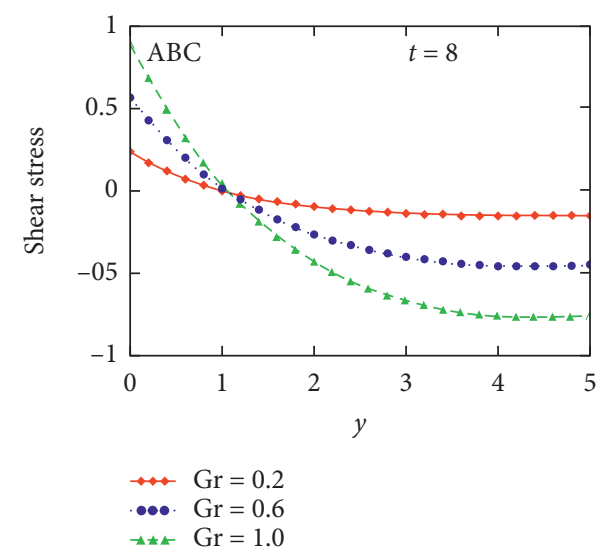

(f)

Figure 6: Shear stress comparison via $\mathrm{C}, \mathrm{CF}$, and $\mathrm{ABC}$ with the variation of the Grashof number $\mathrm{Gr}$, and $\operatorname{Pr}=0.3, a=2$, and $\lambda=0.5$. 

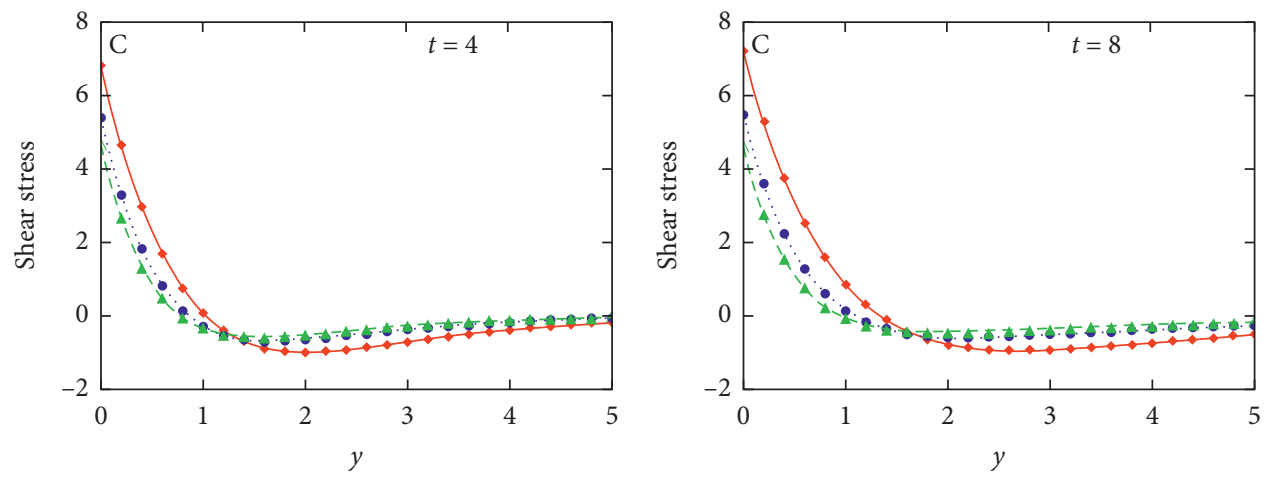

Pr $=5$

•. $\operatorname{Pr}=10$

네나 $\operatorname{Pr}=15$

$$
\begin{array}{ll}
\ldots P & P r=5 \\
\cdots P & P r=10 \\
\leadsto \notin & P r=15
\end{array}
$$

(a)
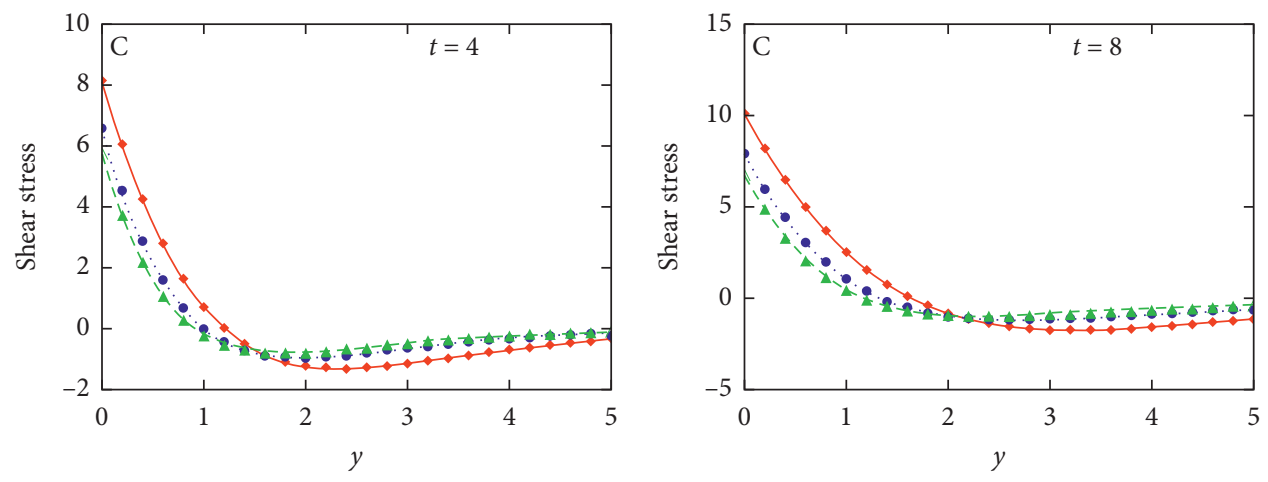

$\rightarrow \quad \operatorname{Pr}=5$

... $\operatorname{Pr}=10$

$\begin{aligned} \operatorname{Pr} & =5 \\ \operatorname{Pr} & =10\end{aligned}$

•.. $\operatorname{Pr}=10$

A\#位 $\operatorname{Pr}=15$

A\#齿 $\operatorname{Pr}=15$

(c)

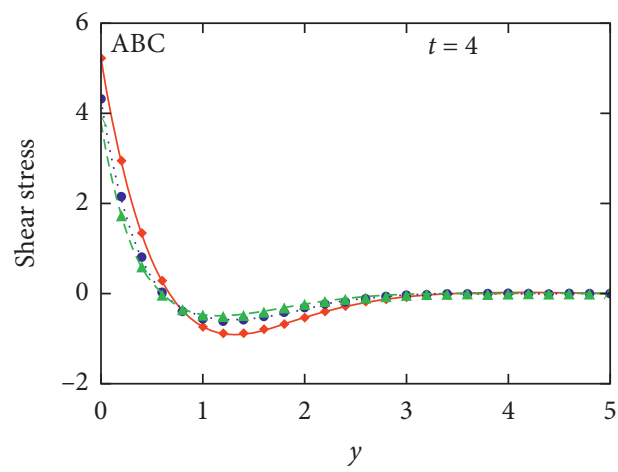

(d)

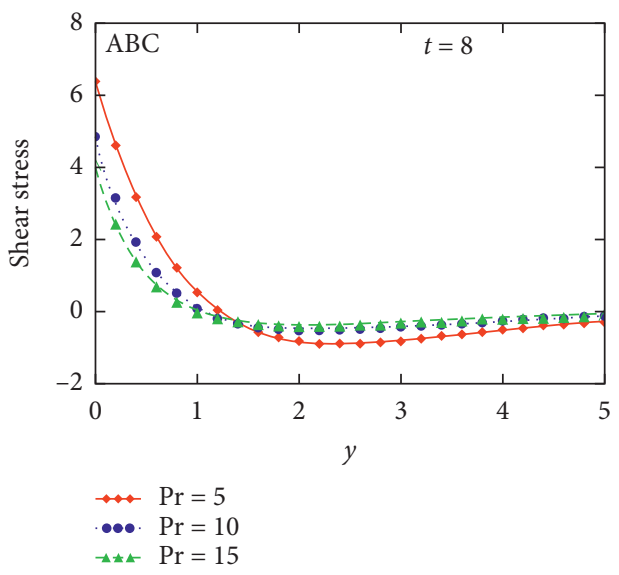

(e)

(f)

FIgURE 7: Shear stress comparison via $\mathrm{C}, \mathrm{CF}$, and $\mathrm{ABC}$ with the variation of the Prandtl number $\operatorname{Pr}$, and $\mathrm{Gr}=10, a=2$, and $\lambda=0.3$. 


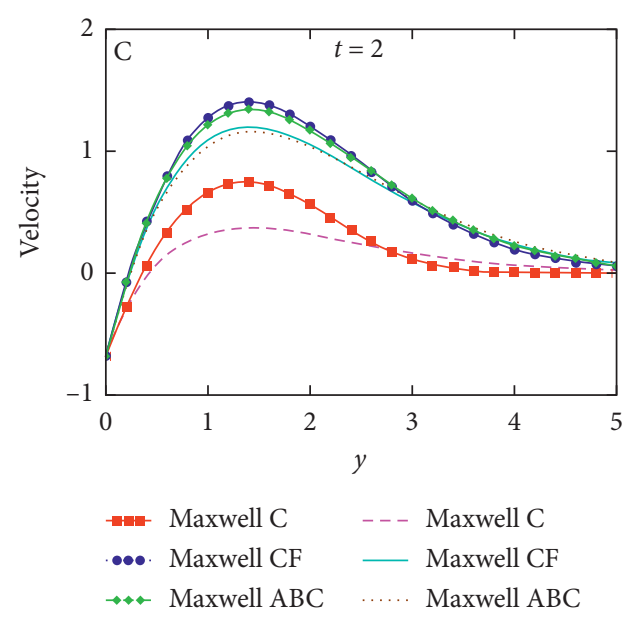

(a)

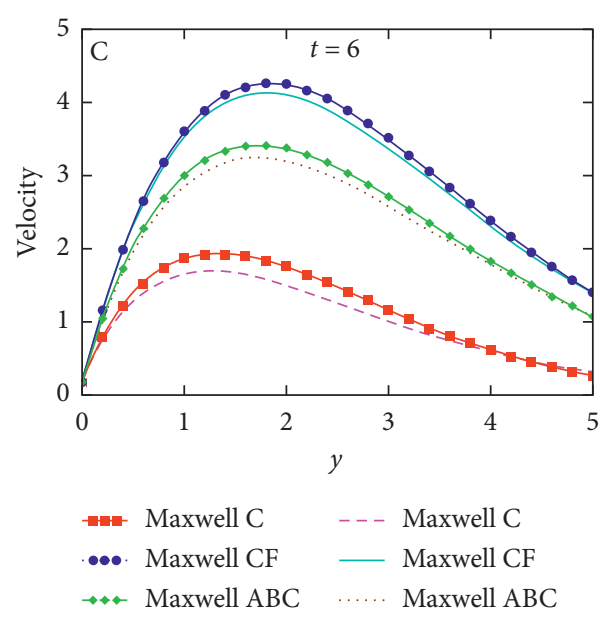

(b)

Figure 8: Velocity comparison via C, CF, and ABC for Maxwell and Newtonian fluids.

\section{Conclusion}

The problem of the heat transfer due to mixed convective fluid flow in the Maxwell fluid is brought into consideration here. The noninteger-order derivative definitions of $\mathrm{C}, \mathrm{CF}$, and $\mathrm{ABC}$ were employed to formulate the problem. The semianalytical solutions were obtained by the Laplace transform and numerical inverse Laplace transform. The comparative analysis of the behavior of the solutions of the velocity, temperature, and shear stress under the influence of different pertinent parameters is brought to light. The following results are concluded from this analysis:

(1) Temperature decreases with the increase in Pr for the variation in time

(2) Velocity increases with the rise of Gr values and declines with the increment in $\mathrm{Pr}$

(3) Shear stress shows the increasing behavior up to a critical point and then decreases under the influence of $\mathrm{Gr}$

(4) Shear stress shows the decreasing behavior up to a critical point and then increases under the impact of $\operatorname{Pr}$

(5) While comparing between the noninteger-order derivative approaches $(\mathrm{C}, \mathrm{CF}$, and $\mathrm{ABC})$, we noticed that the significance of $\mathrm{CF}$ is more due to the nonsingular kernel

(6) For the validation of our results, the limiting cases of $\alpha \longrightarrow 1$ and $\lambda \longrightarrow 0$ were considered, and obtained results were already present in the literature

\section{Data Availability}

All the related data used to support this study are available within the article.

\section{Conflicts of Interest}

The authors declare that they have no conflicts of interest.

\section{Acknowledgments}

The authors appreciate and thank for all the support provided by the respective universities to make this manuscript better.

\section{References}

[1] S.-F. Chou and I.-P. Tsern, "Mixed convection heat transfer of horizontal channel flow over a heated block," Transport Phenomena in Heat and Mass Transfer, vol. 2, pp. 492-503, 1992.

[2] N. Khan, T. Mahmood, M. Sajid, and M. S. Hashmi, "Heat and mass transfer on MHD mixed convection axisymmetric chemically reactive flow of Maxwell fluid driven by exothermal and isothermal stretching disks," International Journal of Heat and Mass Transfer, vol. 92, pp. 1090-1105, 2016.

[3] I. khan, N. A. Shah, and L. C. C. Dennis, "A scientific report on heat transfer analysis in mixed convection flow of Maxwell fluid over an oscillating vertical plate," SCIentIfIC RepoRts, vol. 7, no. 1, Article ID 40147, 2017.

[4] N. Khan, H. A. Nabwey, M. S. Hashmi, S. U. Khan, and I. Tlili, "A theoretical analysis for mixed convection flow of Maxwell fluid between two infinite isothermal stretching disks with heat source/sink," Symmetry, vol. 12, no. 1, p. 62, 2019.

[5] J. C. Maxwell, "IV. On the dynamical theory of gases," Philosophical Transactions of the Royal Society of London, vol. 157, pp. 49-88, 1867.

[6] R. L. Bagley and P. J. Torvik, "A theoretical basis for the application of fractional calculus to viscoelasticity," Journal of Rheology, vol. 27, no. 3, pp. 201-210, 1983.

[7] A. Germnat, "On fractional differentials," Computers and Mathematics with Application, vol. 25, pp. 540-549, 1938.

[8] F. Mainardi and G. Spada, "Creep, relaxation and viscosity properties for basic fractional models in rheology," The European Physical Journal Special Topics, vol. 193, no. 1, pp. 133-160, 2011.

[9] Q. Haito and X. Mingyu, "Unsteady flow of viscoelastic fluid with fractional Maxwell model in a channel," Mechanics Research Communications, vol. 34, no. 2, pp. 210-212, 2007. 
[10] M. Jamil, "Effects of slip on oscillating fractionalized Maxwell fluid," Nonlinear Engineering, vol. 5, no. 1, pp. 25-36, 2016.

[11] I. Khan, F. Ali, U. S. Haq, and S. Shafie, "Exact solutions for unsteady MHD oscillatory flow of a Maxwell fluid in a porous medium," Journal of Nature Research, vol. 68a, pp. 635-645, 2013.

[12] D. Vieru and A. Zafar, "Some Couette flows of a Maxwell fluid with wall slip condition," Applied Mathematics \& Information Sciences, vol. 7, no. 1, pp. 209-219, 2013.

[13] M. A. Imran, M. B. Riaz, N. A. Shah, and A. A. Zafar, "Boundary layer flow of MHD generalized Maxwell fluid over an exponentially accelerated infinite vertical surface with slip and Newtonian heating at the boundary," Results in Physics, vol. 8, pp. 1061-1067, 2018.

[14] S. Aman, Q. Al-Mdallal, and I. Khan, "Heat transfer and second order slip effect on MHD flow of fractional Maxwell fluid in a porous medium," Journal of King Saud University Science, vol. 32, no. 1, pp. 450-458, 2018.

[15] N. Raza and M. A. Ullah, "A comparative study of heat transfer analysis of fractional Maxwell fluid by using Caputo and Caputo-Fabrizio derivatives," Canadian Journal of Physics, vol. 98, no. 1, pp. 89-101, 2019.

[16] J. H. Merkin and T. Mahmood, "Convective flows on reactive surfaces in porous media," Transport in Porous Media, vol. 33, no. 3, pp. 279-293, 1998.

[17] B. J. Minto, D. B. Ingham, and I. Pop, "Free convection driven by an exothermic reaction on a vertical surface embedded in porous media," International Journal of Heat and Mass Transfer, vol. 41, no. 1, pp. 11-23, 1998.

[18] K. A. Abro, I. Khan, and A. Tassaddiq, "Application of Atangana-Baleanu fractional derivative to Convection flow of MHD Maxwell fluid in a porous Medium over a vertical plate," Édition Diffusion Presse Sciences, vol. 13, no. 1, p. 12, 2018.

[19] I. Khan, N. A. Shah, Y. Mahsud, and D. Vieru, "Heat transfer analysis in a Maxwell fluid over an oscillating vertical plate using fractional Caputo-Fabrizio derivatives," The European Physical Journal Plus, vol. 132, no. 4, p. 12, Article ID 194, 2017.

[20] N. A. Shah and I. Khan, "Heat transfer analysis in a second grade fluid over and oscillating vertical plate using fractional Caputo-Fabrizio derivatives," The European Physical Journal C, vol. 76, no. 7, p. 362, 2016.

[21] H. Stehfest's, "Numerical inversion of Laplace transform," Communications of the Association for Computing Machinery, vol. 13, pp. 9-47, 1970.

[22] D. Y. Tzou, Macro to Micro Scale Heat Transfer: The Lagging Behavior, CRC Press, Washington DC, USA, 1997.

[23] C. Fetecau, M. Jamil, C. Fetecau, and I. Siddique, "A note on the second problem of Stokes for Maxwell fluids," International Journal of Non-linear Mechanics, vol. 44, no. 10, pp. 1085-1090, 2009. 